OPEN ACCESS

Edited by:

Bastian Schilling,

University Hospital Würzburg,

Germany

Reviewed by:

Sema Kurtulus,

Novartis Institutes for BioMedical Research, United States

Zachary J. Gerbec,

British Columbia Cancer Research

Centre, Canada

*Correspondence:

William E. Carson

william.carson@osumc.edu

tThese authors have contributed equally to this work

Specialty section: This article was submitted to

Cancer Immunity

and Immunotherapy,

a section of the journal

Frontiers in Immunology

Received: 13 July 2021 Accepted: 23 September 2021 Published: 12 October 2021

Citation:

Sun SH, Benner B, Savardekar H,

Lapurga G, Good L, Abood D,

Nagle E, Duggan $M$, Stiff $A$, DiVincenzo MJ, Suarez-Kelly $L P$, Campbell A, Yu L, Wesolowski R, Howard H, Shah H, Kendra K and Carson WE (2021) Effect of Immune

Checkpoint Blockade on MyeloidDerived Suppressor Cell Populations in Patients With Melanoma. Front. Immunol. 12:740890. doi: 10.3389/fimmu.2021.740890

\section{Effect of Immune Checkpoint Blockade on Myeloid-Derived Suppressor Cell Populations in Patients With Melanoma}

\author{
Steven H. Sun ${ }^{1 \dagger}$, Brooke Benner ${ }^{2 \dagger}$, Himanshu Savardekar ${ }^{2}$, Gabriella Lapurga ${ }^{2}$, \\ Logan Good ${ }^{2}$, David Abood ${ }^{2}$, Erin Nagle ${ }^{2}$, Megan Duggan ${ }^{2}$, Andrew Stiff', \\ Mallory J. DiVincenzo ${ }^{2}$, Lorena P. Suarez-Kelly ${ }^{2}$, Amanda Campbell ${ }^{2}$, Lianbo $\mathrm{Yu}^{3}$, \\ Robert Wesolowski ${ }^{2}$, Harrison Howard ${ }^{2}$, Hiral Shah ${ }^{2}$, Kari Kendra $^{2}$ \\ and William E. Carson ${ }^{1,2 *}$ \\ 1 Department of Surgery, Division of Surgical Oncology, The Ohio State University, Columbus, OH, United States, \\ ${ }^{2}$ Comprehensive Cancer Center, The Ohio State University, Columbus, OH, United States, ${ }^{3}$ Center for Biostatistics, Ohio \\ State University Wexner Medical Center, Columbus, OH, United States
}

Introduction: Myeloid-derived suppressor cells (MDSC) are a subset of immature myeloid cells that inhibit anti-tumor immunity and contribute to immune therapy resistance. MDSC populations were measured in melanoma patients receiving immune checkpoint inhibitors (ICl).

Methods: Patients with melanoma $(n=128)$ provided blood samples at baseline $(B L)$, and before cycles 2 and 3 (BC2, BC3). Peripheral blood mononuclear cells (PBMC) were analyzed for MDSC (CD33 $\left./ \mathrm{CD} 11 \mathrm{~b}^{+} / \mathrm{HLA}-\mathrm{DR}^{\mathrm{lo} /}\right)$ ) and MDSC subsets, monocytic (CD14+, M-MDSC), granulocytic (CD15+, PMN-MDSC), and early (CD14-/CD15-, E-MDSC) via flow cytometry. Statistical analysis employed unpaired and paired t-tests across and within patient cohorts.

Results: Levels of MDSC as a percentage of PBMC increased during ICI (BL: $9.2 \pm 1.0 \%$ to BC3: $23.6 \pm 1.9 \%, p<0.0001$ ), and patients who developed progressive disease (PD) had higher baseline MDSC. In patients who had a complete or partial response (CR, PR), total MDSC levels rose dramatically and plateaued (BL: $6.4 \pm 1.4 \%$, BC2: $26.2 \pm 4.2 \%$, BC3: $27.5 \pm 4.4 \% ; p<0.0001$ ), whereas MDSC rose less sharply in PD patients (BL: $11.7 \pm 2.1 \%$, BC2: $18.3 \pm 3.1 \%$, BC3: $19.0 \pm 3.2 \% ; p=0.1952)$. Subset analysis showed that within the expanding MDSC population, PMN-MDSC and E-MDSC levels decreased, while the proportion of M-MDSC remained constant during $\mathrm{ICl}$. In PD patients, the proportion of PMN-MDSC (as a percentage of total MDSC) decreased (BL: $25.1 \pm 4.7 \%$, BC2: $16.1 \pm$ 5.2\%, BC3: $8.6 \pm 1.8 \%$; $p=0.0105)$, whereas a heretofore under-characterized CD14+ /CD15+ double positive MDSC subpopulation increased significantly (BL: $8.7 \pm 1.4 \%$ to BC3: $26.9 \pm 4.9 \% ; p=0.0425)$. 
Conclusions: MDSC levels initially increased significantly in responders. PMN-MDSC decreased and CD14+CD15+ MDSC increased significantly in PD patients. Changes in MDSC levels may have prognostic value in $\mathrm{ICl}$.

Keywords: nivolumab, pembrolizumab, immune checkpoint blockade, melanoma, MDSC, monocytic MDSC, granulocytic MDSC

\section{INTRODUCTION}

Melanoma accounts for the vast majority of skin cancerassociated deaths, and its incidence has risen rapidly over the past 30 years in the United States. It is estimated that over 95,000 new cases of melanoma will be diagnosed in the United States in 2019 (1). Malignant melanoma has been identified as a tumor type with a high mutational burden (2). Melanoma tumors have been shown to harbor a high number of neoantigens which have the potential to induce a robust host immune response. Patients with advanced melanoma were some of the first to receive immune checkpoint inhibitors and promising efficacy was observed $(3,4)$. This therapeutic approach has revolutionized the treatment of cancer for melanoma and an increasing number of other tumor types (5).

Myeloid-derived suppressor cells (MDSC) are immature myeloid cells with immunosuppressive functions that expand in tumor-bearing hosts in response to tumor-derived factors $(6,7)$. MDSC frequency is increased in patients with cancer including, renal cell carcinoma, hepatocellular carcinoma (HCC), non-small cell lung carcinoma (NSCLC), glioblastoma, gastrointestinal, breast cancer, prostate cancer and melanoma (8-15). Cytokines, chemokines and metabolites produced by tumor cells lead to aberrant myelopoiesis which results in the generation, expansion and recruitment of MDSC to the tumor site. In humans, MDSC are characterized as $\mathrm{CD}_{3} 3^{+}, \mathrm{CD}_{11} \mathrm{~b}^{+}$, and $\operatorname{HLA}_{-} \mathrm{DR}^{\mathrm{lo} / \text { neg }}(6,16,17)$. Further subsets can be defined, with monocytic MDSC (M-MDSC) being characterized as CD14+/CD15-, and granulocytic MDSC (PMN-MDSC) being identified as

CD14-/CD15+. Early MDSC (e-MDSC) are negative for both CD14 and CD15. MDSC are recruited to the tumor by chemokines such as CXCL3, CXCL5, CXCL12, CCL2, and CCL5 where they mediate their immune suppressive effects (18). MDSC can suppress immune cell function utilizing a variety of mechanisms. This includes the generation of reactive oxygen species (ROS) and nitric oxide (NO), the secretion of IL10 and TGF- $\beta$, as well as the over-expression of arginase and IDO, all of which can lead to the inhibition of $\mathrm{T}$ cell function (19). Studies in murine models indicate that disruption of MDSC function can reverse immune tolerance to tumor antigens, stimulate anti-tumor immune responses and improve the efficacy of immune-based therapies such as cancer vaccines and immune checkpoint inhibitors (ICI) $(7,20)$.

Melanoma has been a major focus for the study of cancer immunotherapy due to the occurrence of spontaneous regression in primary tumors, the presence of tumor-infiltrating lymphocytes (21), and the detection of circulating antigenspecific cytotoxic $\mathrm{T}$ cells and antibodies (22). Historically, metastatic melanoma was a disease with an extremely poor prognosis demonstrating a median survival of $<1$ year (23). The advent of immune checkpoint inhibitors directed against PD-1 (nivolumab) and anti-CTLA-4 (ipilimumab) has increased the overall survival of stage III/IV melanoma patients (24). Follow up studies revealed that only a subset of patients (11$34 \%$ ) have a clinically significant or lasting response with monotherapy $(3,25,26)$, sparking the investigation of combinatorial therapy. Results from the CheckMate-067 study showed enhanced survival in patients receiving ipilimumab plus nivolumab compared to either therapy alone at 5 years. However, a significant number of patients (24\%) still did not respond (27).

Since the mechanism behind immunotherapy resistance is not well understood, coupled with the increasing clinical utilization of ICI, there is increasing interest in finding biomarkers that can predict those patients that will respond favorably. Given that the presence of MDSC can attenuate the function of immune cells and potentially inhibit immune-based therapies, strategies that are aimed at depleting or blocking the immune suppressive function of MDSC could be a successful approach to improving the efficacy of checkpoint inhibitors (7, 28). The current study aims to further our understanding of the immunosuppressive milieu in melanoma patients and how it changes with the administration of ICI.

\section{MATERIALS AND METHODS}

\section{Study Design and Patient Population}

The objective of this prospective study was to elucidate the ongoing changes to systemic MDSC populations in patients with advanced melanoma as they receive ICI. Patients with melanoma who were scheduled to receive ICI therapy were considered eligible for this study. There were no specific restrictions with respect to performance status, organ function, or prior/concurrent modes of therapy, or stage of disease. Samples were drawn at the time of initiation of therapy (baseline, BL), and prior to the beginning of cycles 2 (before cycle 2, BC2) and 3 (before cycle 3, BC3) for a total of 3 blood draws. Eligible patients received pembrolizumab, nivolumab, ipilimumab, or nivolumab in combination with ipilimumab. Treatment intervals were dependent on the therapy received: Pembrolizumab was given every 3 weeks, Nivolumab was given every 4 weeks, and combination ipilimumab and nivolumab was given every 3 weeks. Peripheral blood specimens consisted of approximately $50 \mathrm{~mL}$ of blood. Samples were processed as described below. 


\section{Sample Collection and Procurement}

Peripheral blood was obtained from patients with melanoma $(n=128)$ following consent to participate in this IRB-approved prospective clinical study (NCI-2020-06536). Peripheral blood mononuclear cells (PBMC) were isolated from peripheral venous blood via density gradient centrifugation with Ficoll-Paque, as previously described (28). $1 \times 10^{6}$ PBMC were processed and analyzed by flow cytometry as described below.

\section{Assessment of Clinical Response}

Patients were followed in the Melanoma Clinic at the OSU James Cancer Hospital and treatment decisions were made by their oncologist. Response to immunotherapy was assessed using Response Evaluation Criteria in Solid Tumors (RECIST) 1.1.

\section{Flow Cytometry for Myeloid-Derived Suppressor Cells}

PBMC were analyzed for the presence of MDSC as previously described (12). Briefly, MDSC were defined as cells positive for CD33, CD11b and with low to no expression of HLA-DR with subsets expressing CD15 or CD14 representing granulocytic and monocytic MDSC, respectively (Figure 1). Specific antibodies included CD15-FITC, CD33-APC, HLA-DR-PC7, CD11b-PE (all Beckman Coulter), and CD14-V450 (BD Biosciences). All samples were analyzed on a BD LSR II flow cytometer and the data were subsequently analyzed with FlowJo software. Cells were then categorized into specific subsets: early MDSC (CD14-/ CD15-), monocytic MDSC (CD14+/CD15+), granulocytic

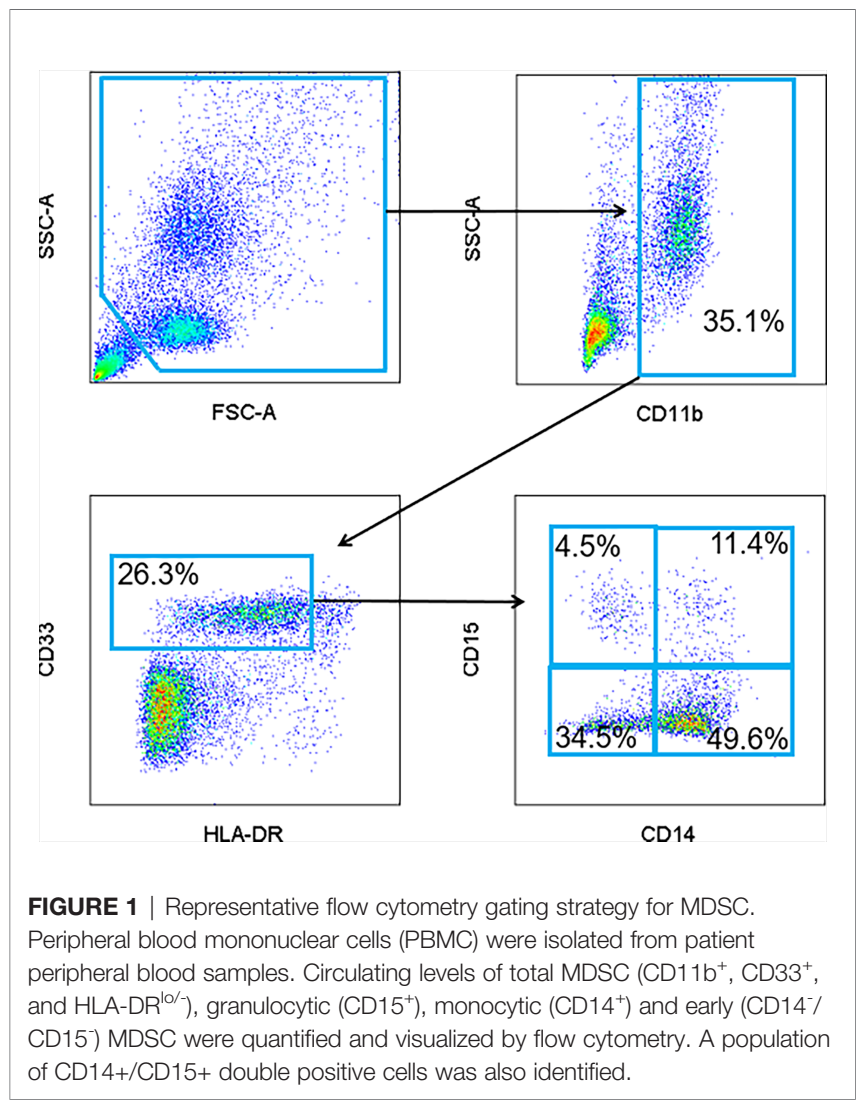

MDSC (CD14-/CD15+). Notably, there is a sizeable population within the gated cells that were double positive (CD14+/CD15+) that are not traditionally described as MDSC. As such, these cells were analyzed separately.

\section{Statistical Analysis}

Statistical analyses were performed using GraphPad Prism Software. Analyses across and between patient cohorts were performed and $\mathrm{p}$ values were generated using unpaired and paired t-tests.

\section{RESULTS}

\section{Patient Characteristics}

A total of 128 patients with melanoma were consented to participate in an IRB-approved prospective clinical registry (OSU-13114). Patients provided blood samples at the initiation of immune checkpoint therapy (before cycle 1 or baseline, BL) and prior to the beginning of cycles 2 (before cycle 2, BC2) and 3 (before cycle 3, BC3). Cycle length was dependent on therapy. Pembrolizumab was given every 3 weeks, nivolumab was every 4 weeks and the combination of ipilimumab and nivolumab was every 3 weeks. Patient characteristics are summarized in Table $\mathbf{1}$. The median age at diagnosis was 60 years and the majority of patients were male $(59 \%, n=75)$. Initial histology subtypes were as follows: superficial spreading $(n=33)$, nodular $(n=28)$, lentigo maligna $(n=13)$, and acral lentiginous melanoma $(n=3)$. Six patients had an otherwise not specified type of melanoma and forty-eight patients were diagnosed at a late stage and did not have primary pathology slides available for confirmation of histology. Forty percent $(n=51)$ of the patients in this cohort had a known BRAF mutation. The majority of patients had measurable metastatic disease $(59 \%, n=75)$, and the rest had locally advanced disease $(41 \%, \mathrm{n}=53)$. Most patients had prior surgery (70\%). Other prior therapy included chemotherapy (6\%), radiation $(13 \%)$, immunotherapy $(31 \%)$, and none (16\%).

\section{Immunotherapy Regimens}

Table 1 summarizes the types of immunotherapy administered. Treatment regimens were chosen by the patient's primary oncologist. Most patients received single agent nivolumab $(n=77)$. Thirty-five patients received pembrolizumab, eleven patients received the combination regimen of ipilimumab and nivolumab, four received ipilimumab. Agent choice was at the physicians' discretion.

\section{Overall Clinical Response in Melanoma Patients Undergoing Immunotherapy for Metastatic Melanoma}

Table 1 also provides a summary of tumor response in the 111 patients who were evaluable for efficacy. Clinical response was evaluated at time of the first restaging scan, most frequently done after 3 cycles of immunotherapy. Overall, 24\% of patients had a complete response (CR), 15\% had a partial response (PR), 23\% had stable disease (SD), and $26 \%$ had progressive disease (PD). 
TABLE 1 | Demographic data for the 128 patients included in the study are listed. Disease characteristics, prior treatment modalities including

immunotherapy and treatment response are included.

Total $\mathbf{N}=128$

\begin{tabular}{|c|c|}
\hline \multicolumn{2}{|l|}{ Age on Dx (years) } \\
\hline Mean & 60 \\
\hline Median & 63 \\
\hline Min, Max & 8,96 \\
\hline SD & 15.32 \\
\hline \multicolumn{2}{|l|}{ Gender, n (\%) } \\
\hline Male & $75(59)$ \\
\hline Female & $53(41)$ \\
\hline \multicolumn{2}{|c|}{ Type of Melanoma, n (\%) } \\
\hline Superficial spreading & $33(26)$ \\
\hline Nodular & $31(24)$ \\
\hline Lentigo maligna & $13(10)$ \\
\hline Acral lentiginous & $3(2)$ \\
\hline Other & $6(5)$ \\
\hline Unknown & $48(38)$ \\
\hline \multicolumn{2}{|l|}{ BRAF Mutation, n (\%) } \\
\hline Yes & $51(40)$ \\
\hline No & $52(40)$ \\
\hline Unknown & $25(20)$ \\
\hline \multicolumn{2}{|l|}{ Metastasis, n (\%) } \\
\hline Yes & $75(59)$ \\
\hline No & $53(41)$ \\
\hline \multicolumn{2}{|l|}{ Prior Treatment, n (\%) } \\
\hline Surgery & $89(70)$ \\
\hline Chemotherapy & $8(6)$ \\
\hline Radiation & $17(13)$ \\
\hline Immunotherapy & $40(31)$ \\
\hline None & $21(16)$ \\
\hline \multicolumn{2}{|l|}{ Immunotherapy, n (\%) } \\
\hline $\mathrm{IFN} \alpha$ & $1(1)$ \\
\hline Ipilimumab & $4(3)$ \\
\hline Ipilimumab/Nivolumab & $11(9)$ \\
\hline Nivolumab & $82(64)$ \\
\hline Pembrolizumab & $30(23)$ \\
\hline \multicolumn{2}{|c|}{ Best Overall Response, n (\%) } \\
\hline Complete Response & $31(24)$ \\
\hline Partial Response & $18(15)$ \\
\hline Stable Disease & $30(23)$ \\
\hline Progressive Disease & $32(27)$ \\
\hline Not Evaluable & $17(13)$ \\
\hline
\end{tabular}

The remaining $13 \%$ of patients could not be assessed, primarily because staging scans had not yet obtained by their oncologist. Notably, of the sixty-eight evaluable patients who received nivolumab, 34\% $(n=23)$ experienced a complete response and $12 \%(n=8)$ experienced a partial response. Of the thirty-five evaluable patients who received pembrolizumab, 17\% $(n=6)$ experienced a complete response, and $29 \%(n=10)$ experienced a partial response. With nivolumab, $78 \%$ achieved stable disease or better based on RECIST criteria. With pembrolizumab, 74\% achieved stable disease or better based on RECIST criteria.

\section{Levels of Circulating Myeloid-Derived Suppressor Cells at Baseline}

To determine the changes in the levels of circulating myeloidderived suppressor cells (MDSC) associated with immunotherapy in melanoma patients, total peripheral blood mononuclear cells (PBMCs) were analyzed prior to cycles 1, 2, and 3 of immunotherapy via flow cytometry. The gating strategy is provided in Figure 1. MDSC were identified as $C D 11 b^{+}$, $\mathrm{CD}^{+}{ }^{+}, \mathrm{HLA}-\mathrm{DR}^{\mathrm{lo} / \mathrm{neg}}$ cells. MDSC subsets were further characterized as CD14+ for M-MDSC and CD15+ for PMN-MDSC. Early MDSC, or E-MDSC, were identified as those that were negative for both CD14 and CD15 (16). A unique population of CD14+/CD15+ double positive cells was also identified and these were included in the MDSC analysis. The characteristics and treatment-induced trajectory of this cell population are discussed at the conclusion of this Results section. At baseline, MDSC comprised $9.2 \pm 1.0 \%$ of all PBMC. A greater proportion of MDSC were classified as M-MDSC at $39.5 \pm 3.2 \%$ compared to $16.4 \pm 2.2 \%$ for PMN-MDSC (Figure 2), and the remaining MDSC were $27.7 \pm 2.4 \%$ E-MDSC. The double positive population comprised the remainder of the gated $\mathrm{CD}_{11 b^{+}}, \mathrm{CD}^{+} 3^{+}, \mathrm{HLA}-\mathrm{DR} \mathrm{R}^{\mathrm{lo} / \mathrm{neg}}$ cells $(13.9 \pm 1.7 \%)$.

Patient MDSC levels were then evaluated according to melanoma histology type (Figures 3A, B). Notably, the levels of total MDSC were lowest at baseline in patients with acral lentiginous melanoma at $3.3 \%$, while other subtypes had approximately $10 \%$ MDSC at baseline (superficial spreading: $9.8 \pm 1.9 \%$; nodular: $10.7 \pm 2.4 \%$; lentigo maligna: $11.6 \pm 2.8 \%$; other: $11.4 \pm 6.3 \%)$. Patients with lentigo maligna melanoma had the lowest levels of PMN-MDSC at $10.1 \pm 3.7 \%$ of the total population but the highest levels of M-MDSC at $51.6 \pm 8.8 \%$, whereas acral lentiginous patients had the highest levels of PMN-MDSC at $32.4 \pm 14.8 \%$ but the lowest levels of M-MDSC at $16.9 \pm 4.6 \%$. Patients with acral lentiginous melanoma had the highest levels of E-MDSC among the 4 skin melanoma types, and patients with extra-dermal melanomas (other) also had high levels of E-MDSC. The differences in MDSC subset proportions between different melanoma subtypes were consistent but did not reach statistical significance. Individual dot plots of this data are visualized in Supplemental Figures 1, 2.

\section{Levels of Circulating Myeloid-Derived Suppressor Cells Following Immune Checkpoint Blockade}

Overall MDSC numbers were calculated as a percentage of PBMC and the proportions of each MDSC subtype were calculated from all $\mathrm{CD}_{11} \mathrm{~b}^{+}, \mathrm{CD}_{3} 3^{+}, \mathrm{HLA}-\mathrm{DR}^{\mathrm{lo} / \mathrm{neg}}$ cells. When assessing the individual MDSC subsets, several trends were identified. The frequency of total MDSC in PBMC significantly increased after two cycles of immunotherapy in the total population of patients (BL: $9.2 \pm 1.0 \%$ to BC3 $23.6 \pm$ $1.9 \%$; $<0.0001)$. An analysis of the subset composition of MDSC showed that the PMN-MDSC population responded differently after immunotherapy with the proportion of PMN-MDSC decreasing significantly over 3 cycles (BL: $16.4 \pm 2.2 \%$ to BC3: $9.1 \pm 2.0 \% ; \mathrm{p}=0.0196)$. Similarly, E-MDSC also decreased after the initiation of immunotherapy (BL: $27.7 \pm 2.5 \%$ to BC3: $21.7 \pm$ $2.3 \% ; \mathrm{p}=0.0392)$. Over this time span, levels of M-MDSC did not display such a striking decrease; their levels remained unchanged throughout the course of immunotherapy (BL: $39.5 \pm 3.2 \%$ to BC3: $38.5 \pm 3.2 \%$ ) (Figure 2). It is important to note that these trends in MDSC subset composition took place in the context of 
A

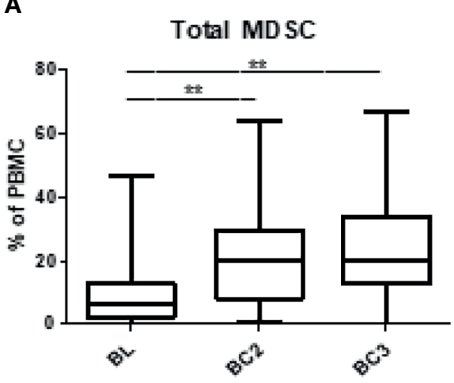

C

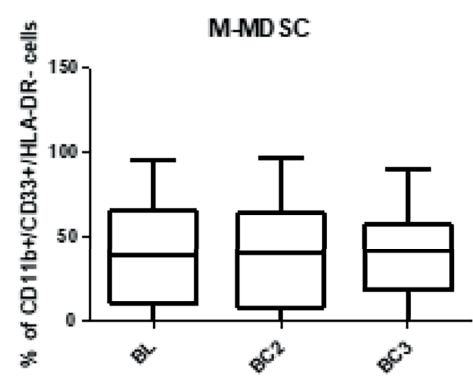

B

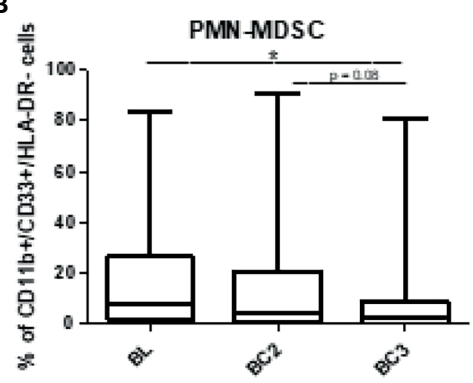

D

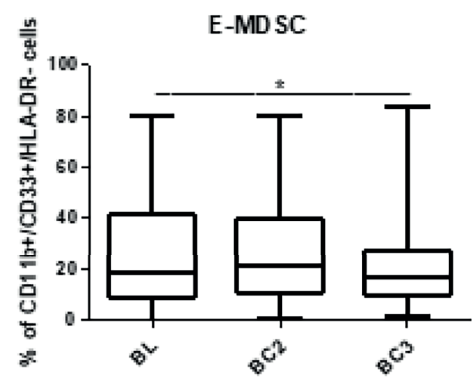

FIGURE 2 | Levels of circulating myeloid-derived suppressor cells over the course of immune checkpoint blockade. Patient PBMCs were isolated at the time of initiation of immune checkpoint therapy (baseline, BL), and prior to the beginning of cycles 2 (before cycle 2, BC2) and 3 (before cycle 3, BC3) and analyzed for total $\operatorname{MDSC}\left(\mathrm{CD} 11 \mathrm{~b}^{+}, \mathrm{CD}_{3}{ }^{+}, \mathrm{HLA}-\mathrm{DR}{ }^{\mathrm{lo} /-}\right)$ and granulocytic $\left(\mathrm{CD} 15^{+}\right)$, monocytic $\left(\mathrm{CD} 14^{+}\right)$, or early (CD14-/CD15-) subsets via flow cytometry. (A) Total MDSC are represented as a percentage of PBMC, and individual subsets represent the proportion of MDSC that fall into each subset: (B) PMN-MDSC, (C) M-MDSC, and (D) E-MDSC Data are shown as box and whisker plots with ranges, ${ }^{\star} p<0.05,{ }^{* *} p<0.01$.

an overall increase in MDSC numbers over three cycles of therapy.

\section{Myeloid-Derived Suppressor Cells and Response to Immunotherapy}

The correlation between levels of MDSC and the patient response to immunotherapy was analyzed. As noted above, the overall frequency of total MDSC in the blood $\left(\mathrm{CD}_{11} \mathrm{~b}^{+}, \mathrm{CD} 33^{+}\right.$, HLA-DR ${ }^{\mathrm{lo} /-}$ ) increased significantly from baseline after just one cycle of immunotherapy in all patients. This effect was maintained after two cycles of immunotherapy in patients with clinical benefit, including those with a complete response (CR; BL: $5.3 \pm 1.1 \%$, BC2: $24.7 \pm 4.2 \%$, BC3: $27.7 \pm 4.7 \%$; $=0.0002)$, partial response (PR; BL: $7.5 \pm 1.6 \%$, BC2: $27.7 \pm 4.2 \%$, BC3: $26.3 \pm 3.9 \% ; \mathrm{p}=0.0024)$, and stable disease (SD; BL: $10.1 \pm 2.9 \%$, BC2: $25.3 \pm 4.3 \%, B C 3: 28.3 \pm 4.0 \% ; \mathrm{p}=0.0138)$. Total MDSC levels also increased in patients who exhibited overall progressive disease, although to a lesser and non-significant extent (PD; BL: $11.7 \pm 2.1 \%$, BC2: $18.3 \pm 3.1 \%$, BC $3: 19.0 \pm 3.2 \%$; $\mathrm{p}=0.1952)$ (Figure 4A).

Changes in circulating levels of $\mathrm{CD}^{+} 5^{+} \mathrm{PMN}-\mathrm{MDSC}$ and $\mathrm{CD}_{1}{ }^{+} \mathrm{M}$-MDSC varied depending on the patient response to immunotherapy. Patients who showed clinical benefit had a significantly lower amount of PMN-MDSC at baseline compared to those with $\mathrm{PD}(\mathrm{CR}+\mathrm{PR}+\mathrm{SD}: 10.7 \pm 1.6 \%$ vs $\mathrm{PD}$ : $26.2 \pm 5.4 \% \mathrm{p}=0.0147)$. PMN-MDSC increased marginally after one cycle of immunotherapy in the clinical benefit population and then decreased to baseline levels after the second cycle of immunotherapy (BL: $10.7 \pm 1.6 \%, \mathrm{BC} 2: 15.3 \pm 2.2 \%$, BC3: $10.2 \pm$ $2.4 \%$ ), whereas PMN-MDSC levels declined at BC2 and BC3 for PD patients (BL: $25.1 \pm 4.7 \%$, BC2: $16.1 \pm 5.2 \%$, BC3: $8.6 \pm 1.8 \%$; $\mathrm{p}=0.0105$ ) (Figure 4B). Indeed, when comparing the four categories of response, the most significant decrease in PMNMDSC after two cycles of immunotherapy was seen in patients with progressive disease $(\mathrm{p}=0.0105)$ (Figure 4C). M-MDSC levels remained largely stable through the course of immunotherapy regardless of response category. There was, however, a significant increase in M-MDSC levels from $30.4 \pm$ $4.8 \%$ at baseline to $48.8 \pm 8.2 \%(\mathrm{p}=0.0392)$ after one cycle of immunotherapy in complete responders, but this effect dissipated after a second cycle of immunotherapy (Figure 4D). Early MDSC trended downward during therapy across all groups but this trend did not reach statistical significance (Figure 4E).

\section{The Effects of Prior Immune Therapy on Circulating MDSC Levels}

The effect of prior immunotherapy on MDSC levels was evaluated. Most of these patients $(n=80)$ had received either IFN- $\alpha$ therapy (26 of 40 ) or cytokine therapy (14 of 40) and none had received prior checkpoint inhibitors. Patients were divided into two groups: those who received prior immunotherapy and those who did not, and their MDSC levels were analyzed prior to 
A Total MDSC by Histology

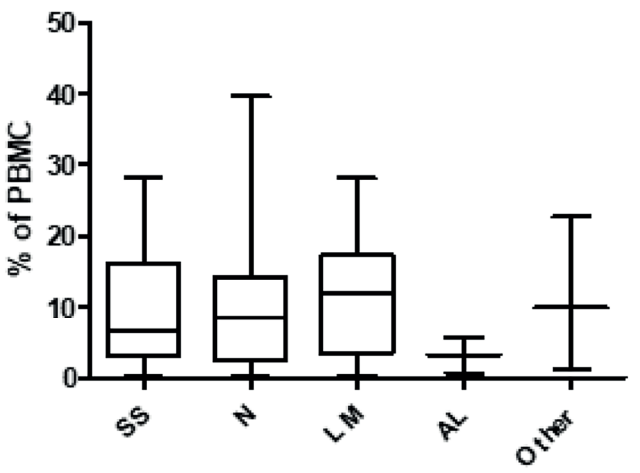

B
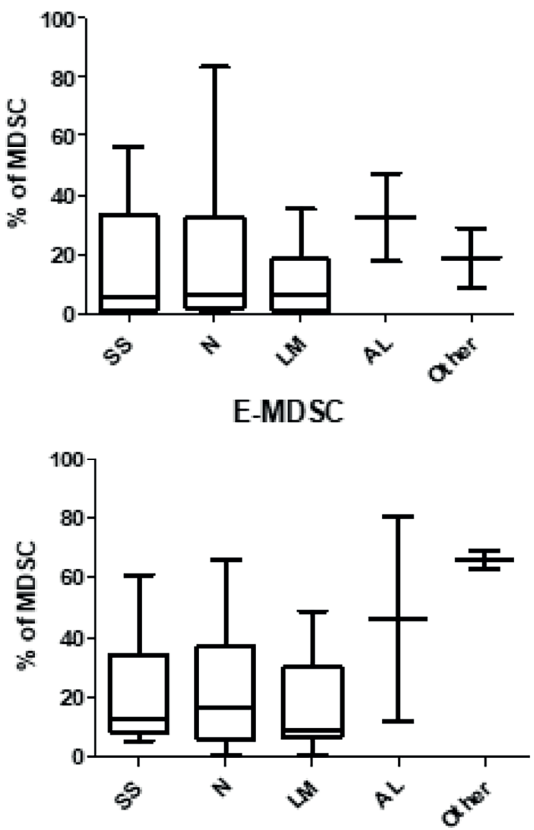

M-MDSC

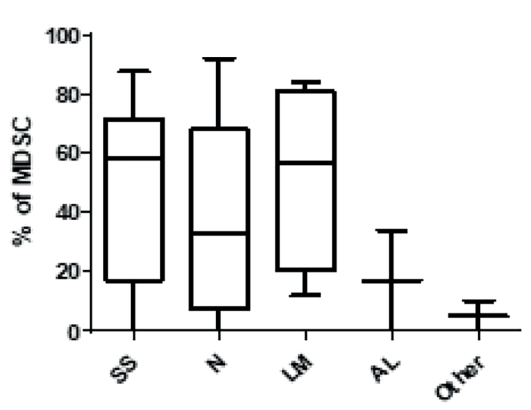

FIGURE 3 | Levels of myeloid-derived suppressor cells according to histologic type of melanoma. Patient PBMCs were isolated from blood draws at the time of initiation of immune checkpoint therapy, and analyzed for MDSC levels and individual subsets. (A) Circulating levels of total MDSC according to histologic type of melanoma. (B) Circulating levels of granulocytic (PMN-MDSC), monocytic (M-MDSC) and early (E-MDSC) MDSC by type of melanoma. SS, Superficial Spreading; N, Nodular; LM, Lentigo Maligna; AL, Acral Lentiginous.

cycles 1, 2, and 3 of current immune checkpoint inhibition. The frequency of total MDSC $\left(\mathrm{CD} 11 \mathrm{~b}^{+}, \mathrm{CD} 33^{+}, \mathrm{HLA}_{-} \mathrm{DR}{ }^{\mathrm{lo} / \mathrm{neg}}\right.$ ) increased across draws 1-3, regardless of whether patients had received prior immunotherapy or not (Figure 5A). Those patients who had previously received immunotherapy exhibited lower levels of PMN-MDSC and higher levels of MMDSC at baseline and after each cycle of therapy. Following initiation of checkpoint inhibition, PMN-MDSC and M-MDSC had very different responses in these two groups (Figures 5B, C). Although circulating levels of PMN-MDSC decreased in both groups of patients, this decrease was significantly pronounced in patients with prior immunotherapy (No prior: BL: $18.5 \pm 4.2 \%$ to BC3 $12.1 \pm 2.2 \%$ vs. prior: BL: $12.3 \pm 2.6 \%$ to BC3 $2.3 \pm 0.6 \%$; $\mathrm{p}=0.0174)$. M-MDSC levels, while elevated in patients with a history of immunotherapy as compared to those with no history (BL: $53.2 \pm 3.6 \%$ vs. $36.1 \pm 2.2 \%, \mathrm{p}=0.0045)$, did not change significantly over the course of/itali treatment (Figure 5C). Early MDSC were higher at all time points in prior immune therapy patients, but levels remained relatively stable in both groups (Figure 5D). 
A

Total MDSC
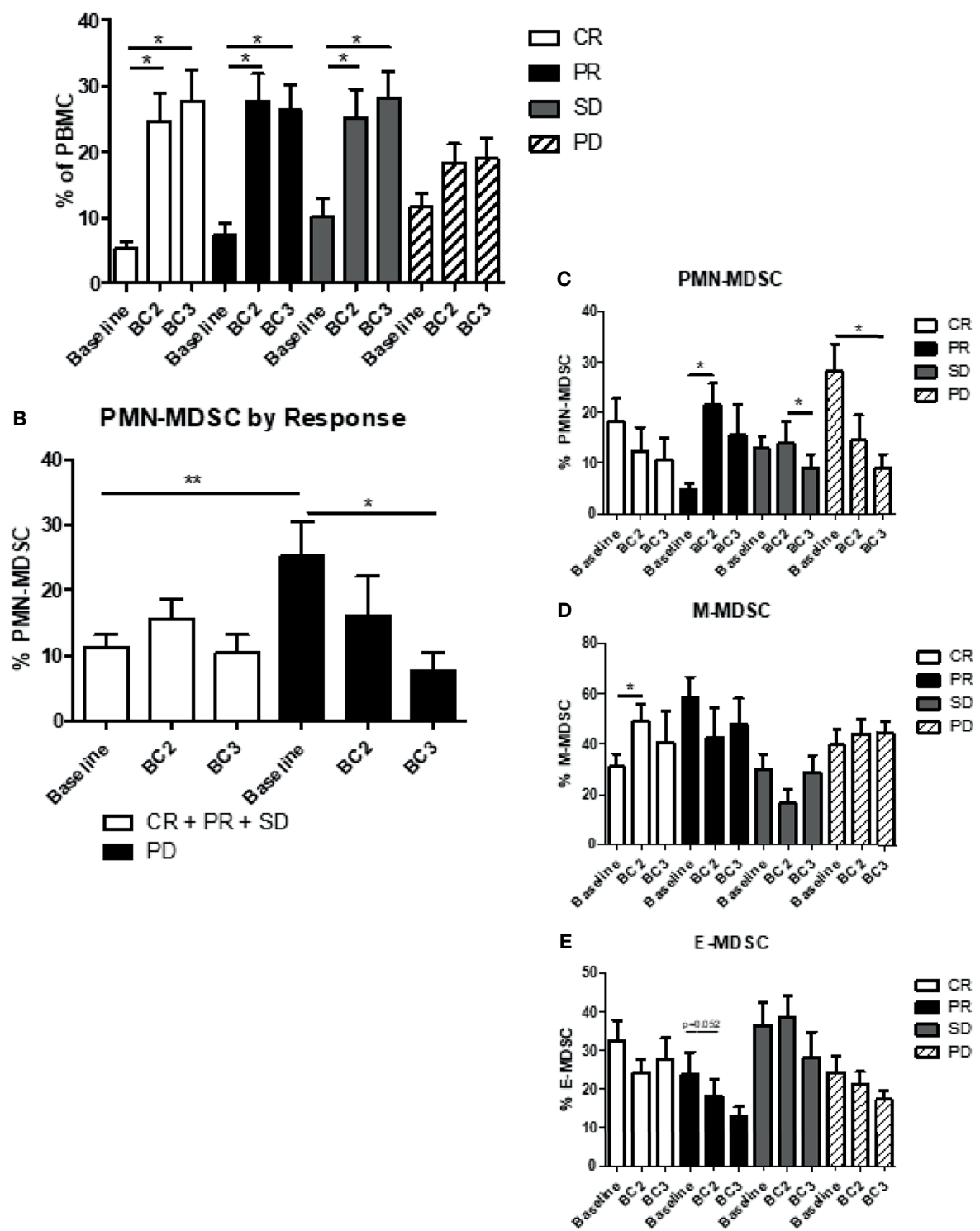

FIGURE 4 | Myeloid-derived suppressor cells and response to immunotherapy. Patient MDSC levels were evaluated according to immunotherapy response, namely clinical benefit (CR + PR + SD) or progressive disease (PD). (A) Levels of total MDSC grouped by patient response to immunotherapy. (B) Levels of granulocytic (PMN-MDSC) in the two response categories are shown. Levels of (C) PMN-MDSC, (D) monocytic MDSC (M-MDSC) and (E) early MDSC (E-MDSC). Data are shown as mean $\pm \mathrm{SE},{ }^{\star} \mathrm{P}<0.05$, ${ }^{\star *} \mathrm{p}<0.01$. CR, complete response; PR, partial response; SD, stable disease; and PD, progressive disease.

\section{Levels of Circulating MDSC Based on the Type of Immunotherapy}

Figures 6A-C summarizes the levels of total MDSC, PMNMDSC, and M-MDSC by type of immune therapy received. Patients were grouped as having received pembrolizumab, nivolumab, ipilimumab and nivolumab in combination, or ipilimumab alone. As noted previously, levels of total circulating MDSC within the PBMC compartment increased over all cycles in all treatment groups except the combination patients. Patients receiving pembrolizumab or nivolumab displayed significant increases in levels of total MDSC after one cycle of immunotherapy. However, PMN-MDSC levels significantly decreased $(12.4 \pm 2.7 \%$ to $2.8 \pm 0.8 \%)$ from baseline after 2 cycles of pembrolizumab. Patients receiving nivolumab also saw decreasing levels of PMN-MDSC from baseline after two cycles but to a lesser extent $(17.8 \pm 2.2 \%$ to $12.7 \pm 3.1 \%$ ) (Figure 6B). M-MDSC levels remained relatively constant across draws 1-3 in patients receiving pembrolizumab, nivolumab or ipilimumab/nivolumab in combination. (Figure 6C). Finally, E-MDSC seemed to decrease with all checkpoint inhibitors with the most significant drop seen in the nivolumab group (Figure 6D). We then correlated treatment 
A Total MDSC

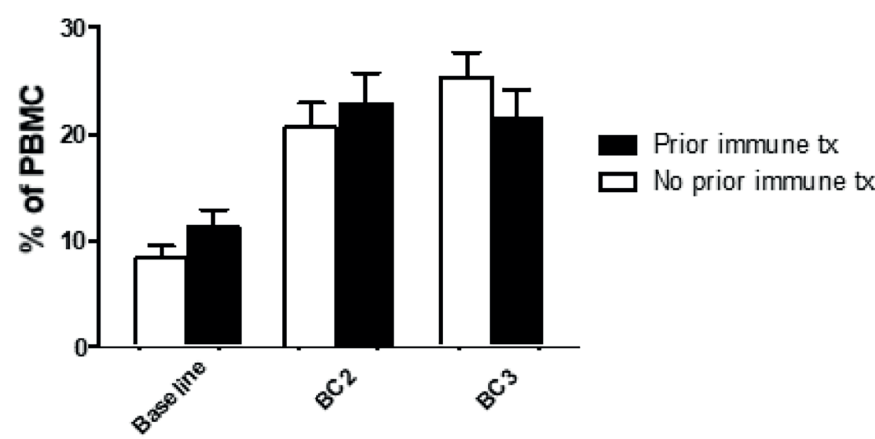

B

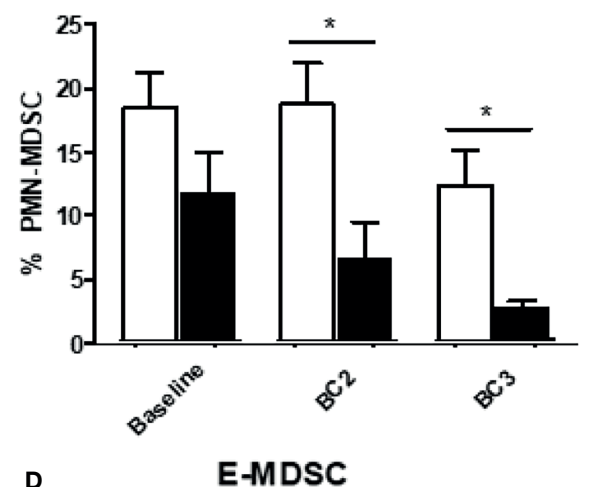

C

M-MDSC

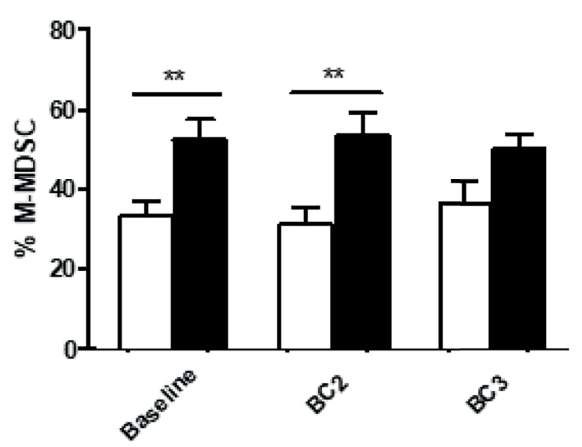

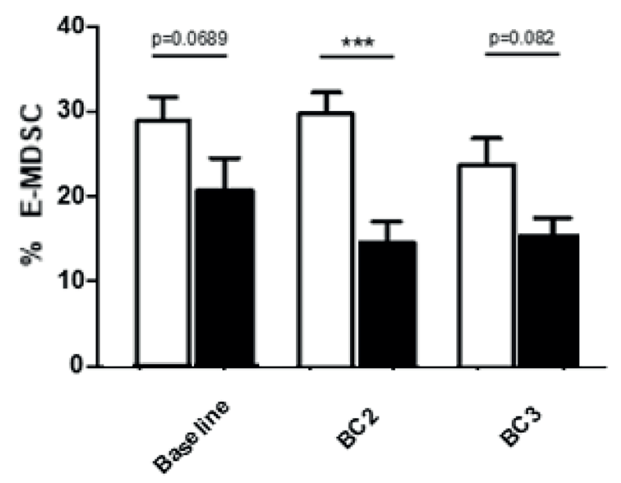

FIGURE 5 | Effects of prior immune system modulation on circulating MDSC levels. Levels of MDSC according to the receipt of prior immunotherapy. Levels of (A) total (B) granulocytic MDSC (PMN-MDSC), (C) monocytic MDSC (M-MDSC), and (D) early MDSC (E-MDSC) are shown. Data are shown as mean \pm SE, ${ }^{*} p<0.05$, ${ }^{* \star} p<0.01,{ }^{* \star *} p<0.001$

responses with the type of immunotherapy regimen given (Figure 6E). Individual dot plots were visualized in Supplemental Figure 3.

\section{Characterization of a CD14+/CD15+ MDSC Subpopulation}

While analyzing the different MDSC subsets, a subpopulation of cells that were positive for both CD14+ and CD15+ was identified. At baseline, these cells represented a small proportion of MDSC, but this proportion significantly increased after checkpoint inhibitor therapy (BL $13.9 \pm 1.7 \%$ vs $\mathrm{BC} 3: 23.3 \pm 2.6 \% ; \mathrm{p}<0.0007)$ (Figure 7A). The proportions of the different CD11b+/CD33
+/HLA-DR ${ }^{\text {lo/neg }}$ subsets are shown over the course of checkpoint inhibition with the size of the pie chart indicating the overall levels of MDSC relative to baseline (Figure 7B). In contrast to the overall trend, in patients who have had previous immune therapy, double positive cells decreased instead of increasing after two cycles of checkpoint inhibition (No prior: $14.2 \pm 2.2 \%$ at $\mathrm{BL}$ to $21.8 \pm 3.3 \%$ at $\mathrm{BC} 3$, prior: $13.3 \pm 2.6 \%$ at $\mathrm{BL}$ to $9.2 \pm 3.6 \%$ at $\mathrm{BC} 3$; $\mathrm{p}=0.0251$ ) (Figure 7C). When grouping by clinical response, we note that progressive patients start with the lowest percentage of these double positive cells, but increase to have the highest percentage by cycle 3 (Figure 7D). When we compared responders versus nonresponders, patients with either complete or partial response had a 


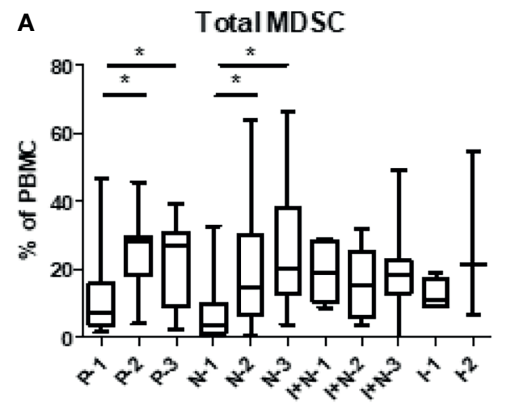

B

PMN-MDSC

$\mathbf{E}$
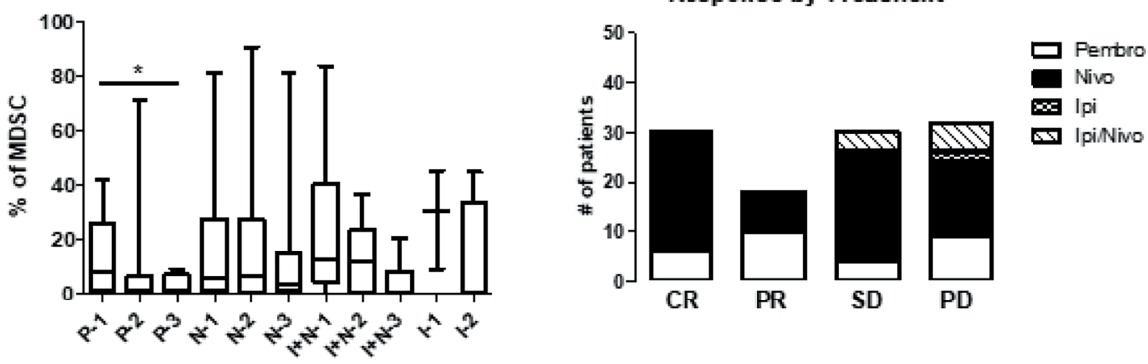

C

M-MDSC

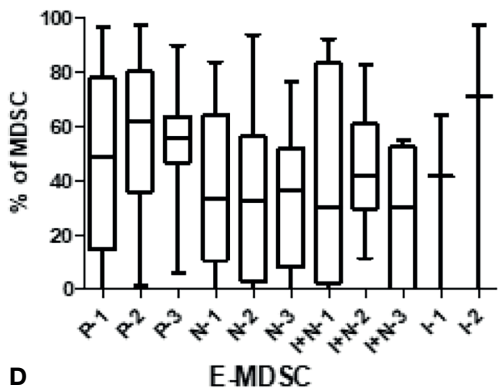

D

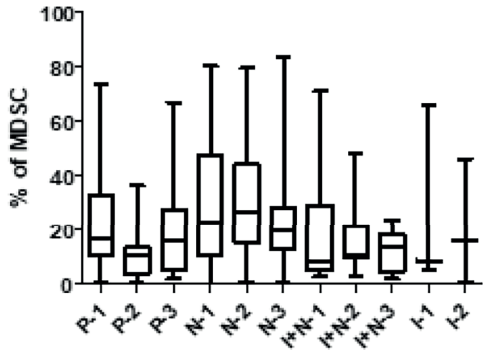

FIGURE 6 | Levels of circulating MDSC based on the type of immunotherapy. Patient PBMCs were evaluated for MDSC levels and analyzed according to type of checkpoint inhibitor therapy received. Patients received either pembrolizumab (Pembro), nivolumab (Nivo), ipilimumab/nivolumab (Ipi/Nivo), or ipilimumab alone (Ipi). Levels of (A) total MDSC (B) granulocytic MDSC (PMN-MDSC), (C) monocytic MDSC (M-MDSC) and (D) early MDSC (E-MDSC) are shown. (E) Clinical response is stratified based on type of immunotherapy given. Data are shown as box and whisker plots with ranges, ${ }^{*} \mathrm{p}<0.05$.

non-significant increase (BL: $14.09 \pm 2.5 \%$ to BL3: $20.38 \pm 4.3 \%$; $\mathrm{p}=0.57$ ), whereas patients with progressive disease had a significant increase (BL: $8.7 \pm 1.4 \%$ to BC3: $26.9 \pm 4.9 \%$; $p=0.0425$ ) (Figure 7E).

\section{DISCUSSION}

The effect of immune checkpoint inhibitor treatment on MDSC populations in patients with advanced melanoma was evaluated.
In this patient population roughly $10 \%$ of PBMC were phenotyped as MDSC at baseline. In addition to these welldescribed MDSC subtypes, a fourth group of cells that were double positive for CD14 and CD15 was identified. Following the initiation of immune checkpoint blockade, overall levels of circulating MDSC increased in melanoma patients. Responders to immunotherapy had MDSC levels that initially rose dramatically and then plateaued. MDSC levels rose to a lesser extent in patients who progressed on immunotherapy. Also, it 
A

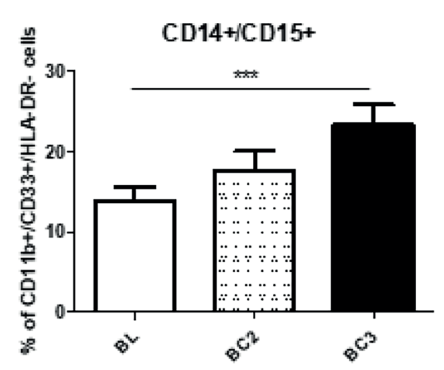

B

BL
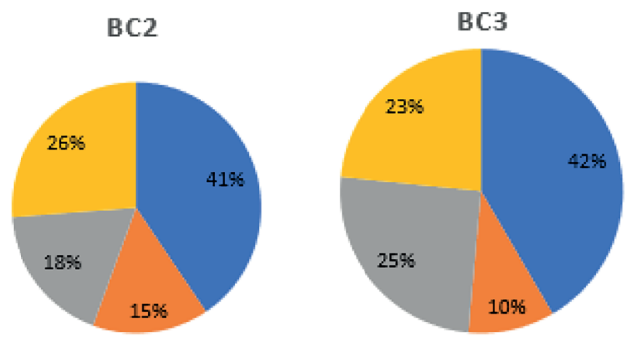

- M-MDSC

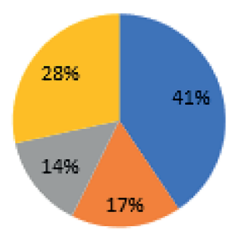

Prior Immune Tx

PMN-MDSC

플 $\mathrm{CD} 14+/ \mathrm{CO} 15+$

II E-MDSC

C
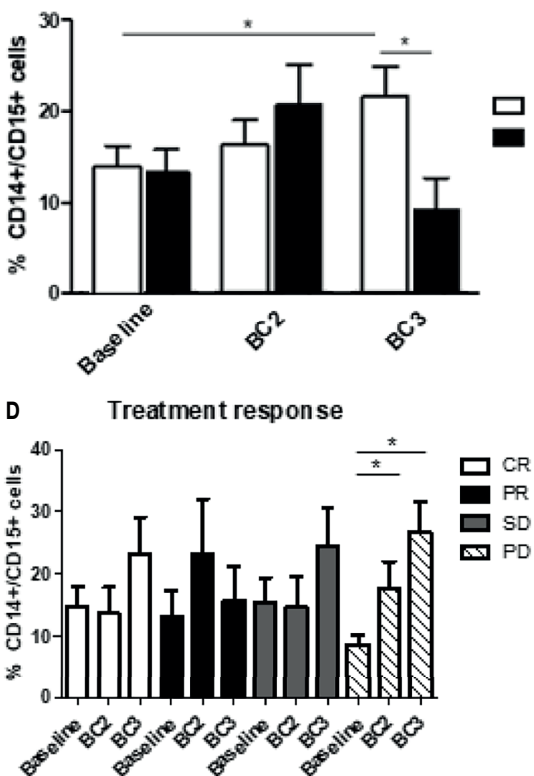

E

Responders vs Non-Responders

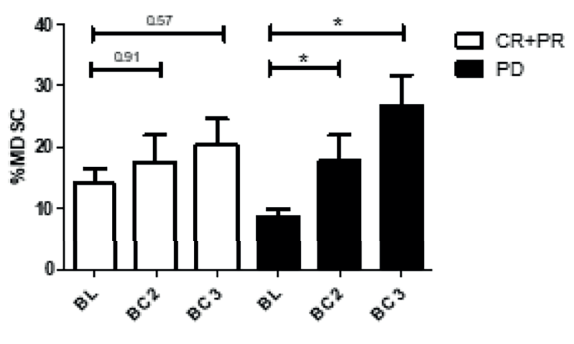

FIGURE 7 | Levels of CD14/CD15 double positive cells. (A) Patient PBMCs were evaluated for CD14/CD15 double positive cells and analyzed over the course of immune checkpoint blockade. (B) Changes to the proportions of the different MDSC subsets along with these double positive cells are shown over the course of immune checkpoint blockade, with size of pie chart corresponding to increasing MDSC percentage. Levels of the double positive cells were analyzed with regards to (C) prior immune therapy, (D) clinical response, (E) responders vs non-responders. Data are shown as mean $\pm \mathrm{SE},{ }^{\star} \mathrm{p}<0.05,{ }^{\star \star *} \mathrm{p}<0.001$.

was observed that patients with progressive disease had the highest baseline level of MDSCs, as well as the smallest increase in MDSC numbers following checkpoint inhibition. When evaluating the proportions of the different MDSC subsets, it was found that M-MDSC were the most prevalent subtype at $41 \%$, followed by E-MDSC at $28 \%$, and PMN-MDSC at $17 \%$. Notably, the various MDSC subsets behaved differently after immune checkpoint blockade. The proportion of PMNMDSC and E-MDSC levels decreased, while the proportion of M-MDSC remained largely constant. At the same time, the proportion of CD14+/CD15 double positive cells increased significantly. In PD patients, the proportion of PMN-MDSC decreased significantly, whereas a heretofore undercharacterized CD14+/CD15+ double positive MDSC subpopulation increased significantly from $8.7 \pm 1.4 \%$ to $\mathrm{BC} 3$ : $26.9 \pm 4.9 \%$; $=0.0425$ ). There were differences in MDSC levels in relation to the histologic subtype of the primary cancer, with the lowest baseline levels being seen in acral lentiginous primaries. These findings further define the characteristics and behavior of this inhibitory immune cell population and provide justification 
for future exploration of their role in modulating the response to immune therapies.

There are multiple mechanisms by which MDSC exert their immunosuppressive effects, and the recruitment of MDSC into tumors represents a distinct mechanism for suppression of the anti-tumor immune response (29). In this study, levels of circulating MDSC in patients with advanced melanoma increased from baseline over the course of three cycles of immunotherapy. The most significant increase was seen immediately after the first dose of ICI, and this effect was sustained over the 3-month time course of the study. The mechanisms underlying this upward MDSC trajectory are not clear. However, it should be noted that systemic levels of immune suppressor cells may not mirror those within the tumor (30). It is also possible that ICI-initiated perturbation of the local tumor microenvironment might lead to the release of cytokines with the ability to induce the generation of MDSC (31). These events might be viewed as evidence of an immunologic "arms race" between malignant cells and activated T cells (32).

Immune checkpoint inhibitors have provided durable responses in patients with advanced-stage melanoma, as well as a variety of other solid tumors. However, a subset of patients become resistant or do not initially respond to immunotherapy (33). One possible contributor to resistance are immune suppressive cells such as MDSC. MDSC have been previously characterized as potential mediators of resistance to ICI (34-36). The different MDSC subsets, granulocytic and monocytic, have been studied and seem to have differential effects in their ability to suppress the $\mathrm{T}$ cell compartment. Levels of granulocytic MDSC (PMN-MDSC) have been shown to consistently repress $\mathrm{T}$ cell responses and correlate with overall survival (15). Studies have shown that higher levels of PMN-MDSC significantly increases the risk of disease progression, which suggests the potential positive effects of targeting MDSC in the context of checkpoint blockade (14). The actual proportion of PMN-MDSC within the expanding MDSC population significantly decreased in this cohort of melanoma patients. The initiation of immune checkpoint blockade resulted in the preferential depletion of PMN-MDSC, and the most significant decrease in PMN-MDSC after two cycles of immunotherapy was seen in patients with progressive disease. Pembrolizumab and nivolumab showed similar effects on MDSC, but with pembrolizumab having a more pronounced effect on PMN-MDSC. In addition, melanoma patients who went on to develop progressive disease following ICI had higher levels of PMN-MDSC at baseline as compared to patients who exhibited clinical benefit. The present data suggests that ICI may have a greater modulatory effect on the PMNMDSC population, which may be independent of the patient's clinical response.

Efforts have been made to find effective biomarkers to help better characterize or predict clinical response to immunotherapy. Martens et al. found that lower levels of LDH and lower levels of CD14+ MDSC correlated with better clinical response to ipilimumab therapy (37). In addition, this group found that the occurrence of adverse events did not correlate with either baseline biomarker signatures or the clinical benefit of ipilimumab. Meyer et al. found that following ipilimumab treatment, MDSC levels remained stable. They reported that responders had overall lower levels of CD14+ MDSC compared to non-responders (28). Similarly, Pico de Coana et al. showed that high levels of classical monocytes and low levels of $\mathrm{M}$ MDSC correlated with improved response rates and overall survival in patients receiving pembrolizumab or nivolumab (38). They also reported that PD-L1 expression in MDSC was significantly increased in patients with shorter progression-free survival and correlated inversely with overall survival. Weber et al. found that high numbers of MDSC were associated with poor survival in patients receiving nivolumab after progressing on ipilimumab (39). The present study showed that patients with progressive disease indeed had higher baseline levels of MDSC. Responders to immunotherapy had MDSC levels that rose dramatically after the start of PD-1 blockade and then plateaued. MDSC levels rose to a lesser extent in patients who progressed on immunotherapy. Notably, levels of CD14+ MMDSC did not change significantly over the course of therapy. The lack of predictive value for M-MDSC in the present study might reflect differences in gating technique or the focus on early treatment time points.

A population of MDSC positive for both CD14 and CD15 was identified and these so-called "double positive" MDSC exhibited the greatest increase in patients who developed progressive disease following the initiation of ICI. Nishimoto et al. showed in a rheumatoid arthritis model that CD14/CD15 double positive cells were seen transiently in the differentiation process from stem cells to monocytes (40). Veglia et al. recently analyzed CD14 expression in murine and human neutrophils, and showed that CD15+ neutrophils with the highest expression of CD14 had significant upregulation of MDSC effector pathways, including IL-6, NO and ROS production, suggesting an "activated" MDSC phenotype (41). Although we observe a similar downward trend in PMN-MDSC in both complete responders and those who have progressive disease, we see a significant increase in the double positive population in non-responders to therapy, compared to those who do show clinical response. This shift in proportions within the MDSC population toward a more potent, activated immunosuppressive phenotype may help explain the difference in clinical response to therapy. These findings suggest that the double positive population could serve as a biomarker for progression of disease on CPI as well as potential target for therapy to overcome resistance. Further study of the immune suppressive capabilities of this cell population in melanoma patients is underway.

The histologic type of melanoma appears to play a role in the quantity and composition of the MDSC population. In the present study, the majority of patients had melanomas of either the superficial spreading or nodular type, with fewer patients presenting with the acral lentiginous and lentigo maligna types. The baseline level of total MDSC were fairly equal across all melanoma histology types, except for the acral lentiginous melanomas, which had notably less total MDSC. 
Observations of an impact of the melanoma primary histology on MDSC patterns has not been previously described and may reflect biologic differences in tumor behavior at the molecular level. Notably, there appears to be distinct variability in the proportion of PMN-MDSC to M-MDSC in tumors arising from different tissues, which indicates that the biology of the primary tumor can have a distinct effect on MDSC generation (42).

In summary, melanoma patients have high levels of MDSC prior to therapy, and levels increase over the treatment course when checkpoint inhibitors are employed. Although total MDSC numbers increased over the course of ICI with PD-1 blocking antibodies, the proportion of PMN-MDSC within the overall population decreased significantly. At the same time, the proportion of double positive (CD14+/CD15+) cells increased over the course of therapy, most significantly in patients who progressed on immunotherapy. Future investigations will identify the tumor factors that govern these changes in MDSC levels.

\section{DATA AVAILABILITY STATEMENT}

The raw data supporting the conclusions of this article will be made available by the authors, without undue reservation.

\section{ETHICS STATEMENT}

The studies involving human participants were reviewed and approved by The Ohio State University Institutional Review Board. The patients/participants provided their written informed consent to participate in this study.

\section{REFERENCES}

1. Siegel RL, Miller KD, Jemal A. Cancer Statistics, 2019. CA Cancer J Clin (2019) 69(1):7-34. doi: 10.3322/caac.21551

2. Galuppini F, Dal Pozzo CA, Deckert J, Loupakis F, Fassan M, Baffa R. Tumor Mutation Burden: From Comprehensive Mutational Screening to the Clinic. Cancer Cell Int (2019) 19:209. doi: 10.1186/s12935-019-0929-4

3. Robert C, Schachter J, Long GV, Arance A, Grob JJ, Mortier L, et al. Pembrolizumab Versus Ipilimumab in Advanced Melanoma. N Engl J Med (2015) 372(26):2521-32. doi: 10.1056/NEJMoa1503093

4. Hodi FS, Chiarion-Sileni V, Gonzalez R, Grob JJ, Rutkowski P, Cowey CL, et al. Nivolumab Plus Ipilimumab or Nivolumab Alone Versus Ipilimumab Alone in Advanced Melanoma (CheckMate 067): 4-Year Outcomes of a Multicentre, Randomised, Phase 3 Trial. Lancet Oncol (2018) 19(11):1480-92. doi: 10.1016/S1470-2045(18)30700-9

5. Queirolo P, Boutros A, Tanda E, Spagnolo F, Quaglino P. ImmuneCheckpoint Inhibitors for the Treatment of Metastatic Melanoma: A Model of Cancer Immunotherapy. Semin Cancer Biol (2019) 59:290-7. doi: 10.1016/ j.semcancer.2019.08.001

6. Gabrilovich DI, Nagaraj S. Myeloid-Derived Suppressor Cells as Regulators of the Immune System. Nat Rev Immunol (2009) 9(3):162-74. doi: 10.1038/nri2506

7. Stiff A, Trikha P, Wesolowski R, Kendra K, Hsu V, Uppati S, et al. 3rd, Myeloid-Derived Suppressor Cells Express Bruton's Tyrosine Kinase and Can Be Depleted in Tumor-Bearing Hosts by Ibrutinib Treatment. Cancer Res (2016) 76(8):2125-36. doi: 10.1158/0008-5472.CAN-15-1490

8. Xin H, Zhang C, Herrmann A, Du Y, Figlin R, Yu H. Sunitinib Inhibition of Stat3 Induces Renal Cell Carcinoma Tumor Cell Apoptosis and Reduces

\section{AUTHOR CONTRIBUTIONS}

Authors SS and BB contributed equally to the manuscript. SS, $\mathrm{BB}$, and WC were involved in study conception and design. Experiments and collection and assembly of data were conducted by SS, BB, HSa, GL, LG, DA, EN, MD, AS, MD, LS-K, and AC. All authors were involved in data analysis and interpretation. Administrative support was provided by SS, and WC. LY provided statistical support and analysis. $\mathrm{HH}, \mathrm{HSh}, \mathrm{KK}$, and WC were involved in the provision of patient samples. SS, BB, LS-K, RW, and WC were involved in manuscript writing. Final approval of the manuscript was provided by all authors.

\section{FUNDING}

This work was supported by the National Institutes of Health Grants T32AI 106704-01A1 (SS) and UM1 CA186712 (WC).

\section{ACKNOWLEDGMENTS}

This work was presented as a poster at the 2020 Virtual Annual Meeting for the American Association of Cancer Research.

\section{SUPPLEMENTARY MATERIAL}

The Supplementary Material for this article can be found online at: https://www.frontiersin.org/articles/10.3389/fimmu.2021.740890/ full\#supplementary-material

Immunosuppressive Cells. Cancer Res (2009) 69(6):2506-13. doi: 10.1158/ 0008-5472.CAN-08-4323

9. Hoechst B, Ormandy LA, Ballmaier M, Lehner F, Krüger C, Manns MP, et al. A New Population of Myeloid-Derived Suppressor Cells in Hepatocellular Carcinoma Patients Induces CD4(+)CD25(+)Foxp3(+) T Cells. Gastroenterology (2008) 135(1):234-43. doi: 10.1053/j.gastro.2008.03.020

10. Srivastava MK, Bosch JJ, Thompson JA, Ksander BR, Edelman MJ, OstrandRosenberg S. Lung Cancer Patients' CD4(+) T Cells are Activated In Vitro by MHC II Cell-Based Vaccines Despite the Presence of Myeloid-Derived Suppressor Cells. Cancer Immunol Immunother (2008) 57(10):1493-504. doi: 10.1007/s00262-008-0490-9

11. Ishida S, Huang E, Zuzan H, Spang R, Leone G, West M, et al. Role for E2F in Control of Both DNA Replication and Mitotic Functions as Revealed From DNA Microarray Analysis. Mol Cell Biol (2001) 21(14):4684-99. doi: 10.1128/ MCB.21.14.4684-4699.2001

12. Mundy-Bosse BL, Lesinski GB, Jaime-Ramirez AC, Benninger K, Khan M, Kuppusamy P, et al. Myeloid-Derived Suppressor Cell Inhibition of the IFN Response in Tumor-Bearing Mice. Cancer Res (2011) 71(15):5101-10. doi: 10.1158/0008-5472.CAN-10-2670

13. Montero AJ, Diaz-Montero CM, Deutsch YE, Hurley J, Koniaris LG, Rumboldt T, et al. Phase 2 Study of Neoadjuvant Treatment With NOV-002 in Combination With Doxorubicin and Cyclophosphamide Followed by Docetaxel in Patients With HER-2 Negative Clinical Stage II-IIIc Breast Cancer. Breast Cancer Res Treat (2012) 132(1):215-23. doi: 10.1007/s10549-011-1889-0

14. Vuk-Pavlović S, Bulur PA, Lin Y, Qin R, Szumlanski CL, Zhao X, et al. Immunosuppressive CD14+HLA-DRlow/- Monocytes in Prostate Cancer. Prostate (2010) 70(4):443-55. doi: 10.1002/pros.21078 
15. Jordan KR, Amaria RN, Ramirez O, Callihan EB, Gao D, Borakove M, et al. Myeloid-Derived Suppressor Cells are Associated With Disease Progression and Decreased Overall Survival in Advanced-Stage Melanoma Patients. Cancer Immunol Immunother (2013) 62(11):1711-22. doi: 10.1007/s00262-013-1475-X

16. Bronte V, Brandau S, Chen SH, Colombo MP, Frey AB, Greten TF, et al. Recommendations for Myeloid-Derived Suppressor Cell Nomenclature and Characterization Standards. Nat Commun (2016) 7:12150. doi: 10.1038/ncomms12150

17. Condamine T, Gabrilovich DI. Molecular Mechanisms Regulating MyeloidDerived Suppressor Cell Differentiation and Function. Trends Immunol (2011) 32(1):19-25. doi: 10.1016/j.it.2010.10.002

18. Sawanobori Y, Ueha S, Kurachi M, Shimaoka T, Talmadge JE, Abe J, et al. ChemokineMediated Rapid Turnover of Myeloid-Derived Suppressor Cells in Tumor-Bearing Mice. Blood (2008) 111(12):5457-66. doi: 10.1182/blood-2008-01-136895

19. Serafini P, Borrello I, Bronte V. Myeloid Suppressor Cells in Cancer: Recruitment, Phenotype, Properties, and Mechanisms of Immune Suppression. Semin Cancer Biol (2006) 16(1):53-65. doi: 10.1016/ j.semcancer.2005.07.005

20. Youn JI, Nagaraj S, Collazo M, Gabrilovich DI. Subsets of Myeloid-Derived Suppressor Cells in Tumor-Bearing Mice. J Immunol (2008) 181(8):5791-802. doi: 10.4049/jimmunol.181.8.5791

21. Wickström S, Lövgren T. Expansion of Tumor-Infiltrating Lymphocytes From Melanoma Tumors. Methods Mol Biol (2019) 1913:105-18. doi: 10.1007/978-1-4939-8979-9_7

22. Chevolet I, Speeckaert R, Schreuer M, Neyns B, Krysko O, Bachert C, et al. Clinical Significance of Plasmacytoid Dendritic Cells and Myeloid-Derived Suppressor Cells in Melanoma. J Transl Med (2015) 13:9. doi: 10.1186/s12967-014-0376-x

23. Kim C, Lee CW, Kovacic L, Shah A, Klasa R, Savage KJ. Long-Term Survival in Patients With Metastatic Melanoma Treated With DTIC or Temozolomide. Oncologist (2010) 15(7):765-71. doi: 10.1634/theoncologist.2009-0237

24. Cooper ZA, Juneja VR, Sage PT, Frederick DT, Piris A, Mitra D, et al. Response to BRAF Inhibition in Melanoma Is Enhanced When Combined With Immune Checkpoint Blockade. Cancer Immunol Res (2014) 2(7):64354. doi: 10.1158/2326-6066.CIR-13-0215

25. Hodi FS, O’Day SJ, McDermott DF, Weber RW, Sosman JA, Haanen JB, et al. Improved Survival With Ipilimumab in Patients With Metastatic Melanoma. N Engl J Med (2010) 363(8):711-23. doi: 10.1056/NEJMoa1003466

26. Topalian SL, Sznol M, McDermott DF, Kluger HM, Carvajal RD, Sharfman WH, et al. Survival, Durable Tumor Remission, and Long-Term Safety in Patients With Advanced Melanoma Receiving Nivolumab. J Clin Oncol (2014) 32(10):1020-30. doi: 10.1200/JCO.2013.53.0105

27. Larkin J, Chiarion-Sileni V, Gonzalez R, Grob JJ, Rutkowski P, Lao CD, et al. FiveYear Survival With Combined Nivolumab and Ipilimumab in Advanced Melanoma. N Engl J Med (2019) 381(16):1535-46. doi: 10.1056/NEJMoa1910836

28. Meyer C, Cagnon L, Costa-Nunes CM, Baumgaertner P, Montandon N, Leyvraz L, et al. Frequencies of Circulating MDSC Correlate With Clinical Outcome of Melanoma Patients Treated With Ipilimumab. Cancer Immunol Immunother (2014) 63(3):247-57. doi: 10.1007/s00262-013-1508-5

29. Ugel S, De Sanctis F, Mandruzzato S, Bronte V. Tumor-Induced Myeloid Deviation: When Myeloid-Derived Suppressor Cells Meet Tumor-Associated Macrophages. J Clin Invest (2015) 125(9):3365-76. doi: 10.1172/JCI80006

30. Najjar YG, Rayman P, Jia X, Pavicic PG, Rini BI, Tannenbaum C, et al. MyeloidDerived Suppressor Cell Subset Accumulation in Renal Cell Carcinoma Parenchyma Is Associated With Intratumoral Expression of IL1 $\beta$, IL8, CXCL5, and Mip-1 $\alpha$. Clin Cancer Res (2017) 23(9):2346-55. doi: 10.1158/1078-0432.CCR-15-1823

31. Yamazaki N, Kiyohara Y, Uhara H, Iizuka H, Uehara J, Otsuka F, et al. Cytokine Biomarkers to Predict Antitumor Responses to Nivolumab Suggested in a Phase 2 Study for Advanced Melanoma. Cancer Sci (2017) 108(5):1022-31. doi: 10.1111/cas.13226

32. Bridge JA, Lee JC, Daud A, Wells JW, Bluestone JA. Cytokines, Chemokines, and Other Biomarkers of Response for Checkpoint Inhibitor Therapy in Skin Cancer. Front Med (2018) 5(351). doi: 10.3389/fmed.2018.00351
33. Lesinski GB, Kondadasula SV, Crespin T, Shen L, Kendra K, Walker M, et al. Multiparametric Flow Cytometric Analysis of Inter-Patient Variation in STAT1 Phosphorylation Following Interferon Alfa Immunotherapy. J Natl Cancer Inst (2004) 96(17):1331-42. doi: 10.1093/jnci/djh252

34. Kim SH, Li M, Trousil S, Zhang Y, Pasca di Magliano M, Swanson KD, et al. Phenformin Inhibits Myeloid-Derived Suppressor Cells and Enhances the Anti-Tumor Activity of PD-1 Blockade in Melanoma. J Invest Dermatol (2017) 137(8):1740-8. doi: 10.1016/j.jid.2017.03.033

35. Sade-Feldman M, Kanterman J, Klieger Y, Ish-Shalom E, Olga M, Saragovi A, et al. Clinical Significance of Circulating CD33+CD11b+HLA-DR- Myeloid Cells in Patients With Stage IV Melanoma Treated With Ipilimumab. Clin Cancer Res (2016) 22(23):5661-72. doi: 10.1158/1078-0432.CCR-15-3104

36. Weber R, Fleming V, Hu X, Nagibin V, Groth C, Altevogt P, et al. MyeloidDerived Suppressor Cells Hinder the Anti-Cancer Activity of Immune Checkpoint Inhibitors. Front Immunol (2018) 9:1310. doi: 10.3389/ fimmu.2018.01310

37. Martens A, Wistuba-Hamprecht K, Geukes Foppen M, Yuan J, Postow MA, Wong P, et al. Baseline Peripheral Blood Biomarkers Associated With Clinical Outcome of Advanced Melanoma Patients Treated With Ipilimumab. Clin Cancer Res (2016) 22(12):2908-18. doi: 10.1158/1078-0432.CCR-15-2412

38. Pico de Coaña Y, Wolodarski M, van der Haar Àvila I, Nakajima T, Rentouli S, Lundqvist A, et al. PD-1 Checkpoint Blockade in Advanced Melanoma Patients: NK Cells, Monocytic Subsets and Host PD-L1 Expression as Predictive Biomarker Candidates. Oncoimmunology (2020) 9(1):1786888. doi: 10.1080/2162402X.2020.1786888

39. Weber J, Gibney G, Kudchadkar R, Yu B, Cheng P, Martinez AJ, et al. Phase I/ II Study of Metastatic Melanoma Patients Treated With Nivolumab Who Had Progressed After Ipilimumab. Cancer Immunol Res (2016) 4(4):345-53. doi: 10.1158/2326-6066.CIR-15-0193

40. Nishimoto N, Murakami M, Ito MN, Saito M, Niwa A, Nakahata T. AB0049 Appearance of CD14+CD15+ Poplulation During the Differentiation From RA-IPS Cells Into Monocytes. Ann Rheumatic Dis (2014) 73(Suppl 2):820-0. doi: 10.1136/annrheumdis-2014-eular.3073

41. Veglia F, Hashimoto A, Dweep H, Sanseviero E, De Leo A, Tcyganov E, et al. Analysis of Classical Neutrophils and Polymorphonuclear Myeloid-Derived Suppressor Cells in Cancer Patients and Tumor-Bearing Mice. J Exp Med (2021) 218(4):1-20. doi: 10.1084/jem.20201803

42. Pico de Coaña Y, Poschke I, Gentilcore G, Mao Y, Nyström M, Hansson J, et al. Ipilimumab Treatment Results in an Early Decrease in the Frequency of Circulating Granulocytic Myeloid-Derived Suppressor Cells as Well as Their Arginase1 Production. Cancer Immunol Res (2013) 1(3):158-62. doi: 10.1158/ 2326-6066.CIR-13-0016

Conflict of Interest: The authors declare that the research was conducted in the absence of any commercial or financial relationships that could be construed as a potential conflict of interest.

Publisher's Note: All claims expressed in this article are solely those of the authors and do not necessarily represent those of their affiliated organizations, or those of the publisher, the editors and the reviewers. Any product that may be evaluated in this article, or claim that may be made by its manufacturer, is not guaranteed or endorsed by the publisher.

Copyright (c) 2021 Sun, Benner, Savardekar, Lapurga, Good, Abood, Nagle, Duggan, Stiff, DiVincenzo, Suarez-Kelly, Campbell, Yu, Wesolowski, Howard, Shah, Kendra and Carson. This is an open-access article distributed under the terms of the Creative Commons Attribution License (CC BY). The use, distribution or reproduction in other forums is permitted, provided the original author(s) and the copyright owner(s) are credited and that the original publication in this journal is cited, in accordance with accepted academic practice. No use, distribution or reproduction is permitted which does not comply with these terms. 